\title{
ASSOCIAÇÃO DO ESTADO NUTRICIONAL COM MARCADORES DE ANEMIA FERROPRIVA EM PRÉ-ESCOLARES ATENDIDOS EM UMA ESF DE PRESIDENTE PRUDENTE-SP
}

Maria Clara Gonçalves Siqueira, Lorrayne Dos Santos Bezerra, Camila Zanini Gimenes de Freitas, Simone de Cássia Casadei Buchalla, Sabrina Alves Lenquiste.

Universidade do Oeste Paulista - UNOESTE, Presidente Prudente, SP. e-mail: sabrina.alves@unoeste.br

\section{RESUMO}

A deficiência de ferro é o motivo de $50 \%$ dos casos de anemia, estando relacionada a uma dieta desequilibrada e a má absorção de nutrientes, atingindo principalmente crianças. $O$ objetivo do presente trabalho foi relacionar as medidas antropométricas e marcadores de anemia ferropriva em crianças atendidas em uma Estratégia de Saúde da Família (ESF). Foram coletadas informações nos prontuários sobre o aleitamento materno, indicadores bioquímicos de anemia e medidas antropométricas recentes. Foram estabelecidas análises de correlação entre as medidas antropométricas e os valores do hemograma, considerando-se nível de significância estatística de $p<0,05$. Os resultados demonstram que $27,27 \%$ se encontram em quadro de anemia, sendo que apenas $19,69 \%$ das 66 crianças tinham exames de ferro e ferritina. Não houve correlação entre índice de massa corporal e os resultados de hemoglobina e hematócrito. Conclui-se que a prevalência de anemia ferropriva, identificada pelos valores de hemoglobina foi baixa. Contudo, nota-se elevada ocorrência de suplementação profilática com sulfato ferroso sem indicação, elevando o risco de suplementação excessiva de ferro.

Palavras-chaves: antropometria, crianças, ferro, anemia, suplementação de ferro.

\section{ASSOCIATION OF NUTRITIONAL STATUS WITH MARKERS OF IRON DEFICIENCY ANEMIA IN PRESCHOOL CHILDREN ATTENDING AN ESF IN PRESIDENTE PRUDENTE-SP}

\begin{abstract}
Iron deficiency is the cause of $50 \%$ of anemia cases, being related to an unbalanced diet and nutrient malabsorption, affecting mainly children. The aim of the present study was to relate anthropometric measurements and markers of iron deficiency anemia in children attending a Family Health Strategy (FHS). Information was collected from medical records about breastfeeding, biochemical indicators of anemia and recent anthropometric measurements. Correlation analyzes were established between anthropometric measurements and blood count values, considering a level of statistical significance of $p<0.05$. The results show that $27.27 \%$ are in anemia, and only $19.69 \%$ of the 66 children had iron and ferritin tests. There was no correlation between body mass index and hemoglobin and hematocrit results. It was concluded that the prevalence of iron deficiency anemia, identified by hemoglobin values, was low. However, there is a high occurrence of prophylactic supplementation without indication with ferrous sulfate, increasing the risk of excessive iron supplementation.
\end{abstract}

Keywords: anthropometry, children, iron, anemia, iron supplementation. 


\section{INTRODUÇÃO}

Tem-se como fator de vulnerabilidade para a saúde da criança o período entre o desmame e os cinco anos de vida. $O$ crescimento acelerado, a perda de imunidade passiva e a construção de um sistema imunológico adequado contra possíveis infecções determinam necessidades nutricionais específicas, sendo primordial monitorar o estado nutricional nessa faixa etária ${ }^{1}$. É por essa razão que se torna indispensável o acompanhamento do estado nutricional de crianças, principalmente nos países em desenvolvimento, para identificação precoce de qualquer risco nutricional e a promoção de ações que proporcionem a melhora e recuperação do estado nutricional e saúde global da população infantil ${ }^{2}$.

A alimentação adequada é um elemento que causa grande impacto na saúde infantil, uma vez que é considerada um pré-requisito imprescindível para a promoção, proteção e manutenção da saúde ${ }^{3}$. É durante a infância que a criança forma seu hábito alimentar, o qual o acompanhará durante as demais fases da vida, e, portanto, as escolhas alimentares dos pais impactam qualitativamente e quantitativamente no comportamento alimentar da criança ${ }^{4}$. Nas últimas décadas, a transição alimentar é evidente, sendo caracterizada pela ingestão elevada de alimentos ricos em gordura, açúcar e sódio e menor consumo de verduras, frutas e legumes, propiciando um aumento na obesidade infantil e aparecimento de deficiências nutricionais ${ }^{5}$.

A ingestão insuficiente de alimentos in natura, especialmente frutas, verduras e legumes, compromete o aporte de micronutrientes podendo ter relação negativa no sistema imunológico das crianças frentes a infecções, além de aumentar a ocorrência de deficiências nutricionais ${ }^{6}$. Entre os micronutrientes responsáveis pelo desenvolvimento saudável da criança e seu adequado crescimento, tem-se o ferro, a vitamina $A$ e o zinco como elementos mais importantes. Segundo a literatura, dentre os principais agravos causados pela deficiência desses micronutrientes, encontram-se a diminuição do sistema cognitivo, alterações da imunidade do indivíduo, maior susceptibilidade a infecções e funcionamento inadequado do metabolismo ${ }^{7}$.
A anemia é hoje um problema de saúde de grande magnitude, afetando não somente os países em desenvolvimento, mas também os desenvolvidos. Tratando-se de um problema global, cuja relevância está diretamente associada ao desenvolvimento humano, social e econômico da humanidade como um todo ${ }^{8}$.

A Organização Mundial da Saúde (OMS) define anemia ferropriva como sendo a baixa concentração de hemoglobina no sangue, utilizando como parâmetro os índices considerados normais. A principal causa da doença está associada à deficiência de ferro ${ }^{9}$ e, apesar da mesma poder ser isolada, ela geralmente se apresenta interligada, coexistindo em uma grande variedade de fatores e tendo como resultado final a doença. Portanto, para o diagnóstico da anemia é de fundamental importância à compreensão da complexidade das causas e fatores existentes.

A deficiência de ferro é responsável por $50 \%$ dos casos de anemia. A carência deste micronutriente pode ser resultado de uma baixa ingestão, fruto de uma dieta desequilibrada e/ou má absorção, ou ainda derivada de diversos fatores intrínsecos ao indivíduo. Outro fator relevante para o problema consiste justamente na alta necessidade de ferro em certos períodos da vida, como na gestação e na infância. Consequentemente, temos dois grandes grupos naturalmente mais suscetíveis à doença; mulheres grávidas e crianças pequenas. Diante da complexidade do problema exposto, para se chegar a um diagnóstico e tratamento efetivo, deve-se observar a pluralidade dos fatores de risco $\mathrm{e}$ as particularidades do paciente envolvido ${ }^{8}$.

Alguns fatores de risco podem influenciar diretamente e colaborar para o aparecimento de anemia ferropriva, como por exemplo, a idade materna, havendo maior probabilidade que mães adolescentes tenham filhos com baixo peso, fator este expressamente ligado à menor reserva de hemoglobina ${ }^{10}$. A renda familiar também pode ser considerada um fator importante para a incidência da doença, visto que famílias de baixa renda apresentam maior ocorrência de anemia ferropriva devido ao papel que a renda exerce sobre a possibilidade de aquisição e utilização de serviços e bens essenciais à manutenção da saúde $^{11}$. O aleitamento materno aparece como fator protetor contra o aparecimento da doença, uma vez que o desmame precoce (antes dos 6 
meses de vida) está intimamente ligado à redução da disponibilidade de ferro, diminuindo assim a oferta desse nutriente ao lactente ${ }^{12}$.

Uma das consequências da carência de ferro é a diminuição do transporte de oxigênio, fundamental para a respiração aeróbica, devido aos baixos níveis de hemoglobina que, por sua vez, é a responsável pela entrega do oxigênio aos tecidos $^{13}$. Também possui efeito negativo no crescimento e na puberdade, afetando o desenvolvimento cognitivo, motor e comportamental $^{14}$. Relacionam-se ainda com a deficiência de ferro alterações no sistema imunológico, elevando a morbidade por infecções. Como principais sintomas têm-se a fraqueza, o cansaço, a desatenção social e diminuição do desempenho escolar ${ }^{15}$. Além disso, o ferro é um nutriente essencial para o desenvolvimento e crescimento celular dos sistemas neural e imune ${ }^{16}$. Portanto, a anemia ferropriva, mesmo quando leve ou moderada, é um importante fator que aumenta os riscos de morbidade e mortalidade em crianças, tornando necessário o tratamento e o acompanhamento para evitar complicações futuras ${ }^{8-17}$.

As maiores taxas de deficiência de ferro e anemia ferropriva são apresentadas pelos países com baixa renda, devido à pouca biodisponibilidade de ferro proveniente da alimentação, associada a uma redução de absorção deste nutriente, além de altos níveis de infecção que limitam cronicamente a absorção de ferro. Para suprir as deficiências de ferro a OMS recomenda a implementação de Programas de Suplementação Universal de Ferro para crianças pequenas e mulheres grávidas ${ }^{16}$.

Várias ações têm sido adotadas no Brasil, dentre elas a suplementação profilática com sulfato ferroso. Outrossim, o Ministério da Saúde adotou desde 2005 o Programa Nacional de Suplementação de Ferro (PNSF), que objetiva a prevenção, combate e controle da anemia. O PNSF fundamenta-se na suplementação profilática de ferro para todas as crianças de 6 a 24 meses de idade, gestantes ao iniciarem o prénatal, independentemente da idade gestacional até o terceiro mês pós-parto ${ }^{18}$. O PNSF indica que a dosagem para crianças de 6 a 24 meses seja de $1 \mathrm{mg} / \mathrm{kg}$ peso/dia ${ }^{19}$ com o objetivo de atender as necessidades de ferro diárias para obter resultados no crescimento e desenvolvimento adequados, visto que o ferro obtido da ingestão de alimentos não é suficiente para atender as necessidades exigidas pelo organismo ${ }^{20}$.
Com o objetivo de ampliar o escopo das ações de Atenção Básica, a partir de 2008 foram criados os Núcleos de Apoio à Saúde da Família (NASF), que se caracterizam pela constituição em equipes multiprofissionais, pertencentes à Estratégia Saúde da Família (ESF), atuantes no âmbito da saúde e assistência social, sendo o nutricionista um dos profissionais que pode fazer parte delas. As ações de alimentação e nutrição no âmbito da Atenção Básica visam a ampliação da qualidade dos planos de intervenção, em especial às doenças e agravos não-transmissíveis, no crescimento e desenvolvimento na infância, na gestação e no período de amamentação, evidenciando que a promoção de práticas alimentares saudáveis é importante em todas as fases da vida ${ }^{21,22}$.

Sabe-se que alimentação é o principal meio para se obter ferro, sendo imprescindível uma dieta de qualidade e quantidades adequadas para suprir as necessidades exigidas pelo organismo ${ }^{23}$ e que a suplementação profilática de ferro se dá apenas nas crianças até os 24 meses, sendo que as crianças em idade pré-escolar ( 3 a 6 anos) estão expostas ao maior risco de anemia. Sabe-se ainda que a anemia é um importante fator de prejuízo do crescimento em crianças. Assim, o objetivo do presente estudo foi relacionar fatores de risco de anemia, as medidas antropométricas e marcadores de anemia ferropriva em pré-escolares atendidos em uma ESF de Presidente Prudente - SP.

\section{METODOLOGIA}

A coleta dos dados foi realizada nos meses de agosto a novembro do ano de 2018, seguindo as normas da Resolução CNS 466/2012 do CONEP, conduzida em acordo com os princípios éticos de não maleficência, beneficência, justiça e autonomia contidas na resolução 196/96 do Conselho Nacional de Saúde $^{24}$, sendo registrada na Plataforma Brasil sob o número C.A.A.E. 91166118.3.0000.5515 e aprovada pelo Comitê de Ética em Pesquisa da Universidade do Oeste Paulista.

Foram escolhidos prontuários de crianças em idade pré-escolar ( 3 anos a 6 anos completos) atendidas na Estratégia de Saúde da Família (ESF) De um bairro de Presidente Prudente - SP. Foram inclusas na pesquisa crianças de ambos os sexos, com idade entre 3 a 6 anos, acompanhadas pela equipe de saúde da ESF do bairro, sem nenhuma doença conhecida, com ou sem diagnóstico de anemia ferropriva, cujos prontuários continham 
informações de hemograma completo (hemoglobina - Hb; hematócrito - Ht; e Volume Corpuscular Médio - VCM) e, eventualmente, dosagens de ferro e ferritina dos últimos 6 meses, bem como medidas de peso e altura aferidos em consulta de puericultura nos últimos 6 meses.

As medidas antropométricas de peso e estatura foram utilizadas para realizar a classificação do estado nutricional das crianças, por meio das curvas de crescimento da Organização Mundial de Saúde ${ }^{25}$. Os indicadores avaliados foram: peso para idade $(\mathrm{P} / \mathrm{I})$, estatura para a idade $(E / I)$, peso para a estatura $(P / E)$ e índice de massa corporal - IMC - para a idade (IMC/I), para os quais foi estabelecido o valor de escore $Z$ e classificado o estado nutricional de acordo com o indicador.

Os resultados foram tabulados e apresentados como média \pm desvio-padrão. Foram estabelecidas correlações entre o Índice de Massa Corporal (IMC) e os valores séricos de hemoglobina e hematócrito por meio de regressão linear simples, considerando-se como significativo valor de $p<0,05$.

\section{RESULTADOS}

Da amostra total de 66 crianças que participaram da pesquisa, $33(50 \%)$ pertenciam ao sexo feminino, e $33(50 \%)$ ao sexo masculino. Poucas crianças possuíam os exames bioquímicos referentes à dosagem de ferro e ferritina (Tabela 1). No que se refere aos valores de Hemoglobina sérica $(\mathrm{Hb})$, Hematócrito $(\mathrm{Ht})$ e Volume Corposcular Médio (VCM) a análise dos dados permite verificar que, a média dos resultados se encontra dentro dos valores de referência utilizados como parâmetro para esse estudo (Hemoglobina > 11,5 g/dL), (Hematócrito > 34\%) e (VCM > 75fL). É importante ressaltar que, para que o diagnóstico de anemia seja completamente validado, deve haver uma diminuição comum entre esses fatores.

Tabela 1. Caracterização dos participantes, medidas antropométricas e valores da série vermelha do hemograma (média e desvio-padrão).

\begin{tabular}{lcc}
\hline & Feminino & Masculino \\
\cline { 2 - 3 } 3 a 4 anos e 11 meses & $13(39,39 \%)$ & $18(56,25 \%)$ \\
Peso $(\mathrm{Kg})$ & $17,46 \pm 3,98$ & $16,26 \pm 2,25$ \\
Altura $(\mathrm{cm})$ & $102,08 \pm 9,43$ & $101,00 \pm 3,84$ \\
$\mathrm{IMC}\left(\mathrm{Kg} / \mathrm{m}^{2}\right)$ & $16,64 \pm 2,23$ & $15,90 \pm 1,61$ \\
$\mathrm{Hb}(\mathrm{g} / \mathrm{dL})$ & $12,31 \pm 0,64$ & $11,88 \pm 0,88$ \\
$\mathrm{Ht}(\%)$ & $37,23 \pm 1,73$ & $36,09 \pm 2,31$ \\
VCM $\left(\mu^{3}\right)$ & $79,31 \pm 3,41$ & $76,77 \pm 6,33$ \\
Dosagem de ferro e ferritina & $2(15,40 \%)$ & $6(33,33 \%)$ \\
& Feminino & Masculino \\
\cline { 2 - 3 } 5 a 6 anos e 11 meses & $20(60,61 \%)$ & $14(43,75 \%)$ \\
Peso $(\mathrm{Kg})$ & $22,66 \pm 5,12$ & $20,41 \pm 4,61$ \\
Altura $(\mathrm{cm})$ & $113,5 \pm 6,14$ & $112,43 \pm 5,05$ \\
IMC $\left(\mathrm{Kg} / \mathrm{m}^{2}\right)$ & $17,56 \pm 3,27$ & $16,08 \pm 3,28$ \\
Hb $(\mathrm{g} / \mathrm{dL})$ & $12,83 \pm 0,82$ & $12,44 \pm 0,89$ \\
Ht $(\%)$ & $38,83 \pm 2,35$ & $37,80 \pm 2,97$ \\
VCM $\left(\mu^{3}\right)$ & $81,64 \pm 4,26$ & $78,84 \pm 5,13$ \\
Dosagem de ferro e ferritina & $2(10,00 \%)$ & $3(23,08 \%)$ \\
\hline
\end{tabular}

$\mathrm{IMC}=$ Índice de Massa Corporal; $\mathrm{Hb}=$ Hemoglobina; $\mathrm{Ht}=$ Hematócrito; VCM = Volume Corpuscular Médio.

Na Tabela 2 são observados os resultados referentes ao diagnóstico de anemia e suplementação de ferro (sulfato ferroso). A partir dos valores de referência para o hemograma, descritos acima, foi possível identificar a 
ocorrência de anemia nas crianças. Nota-se nesta tabela que, apesar da baixa prevalência de anemia nessa população, mais da metade das crianças, independente da faixa etária, recebiam suplementação com sulfato ferroso, mesmo sem indicação de suplementação profilática para essa idade.
A informação a respeito do aleitamento materno, a qual deve ser registrada pela equipe de saúde, foi obtida para cerca de $47 \%$ das crianças estudadas. A Figura 1 mostra a distribuição das informações sobre o aleitamento materno nas crianças estudadas.

Tabela 2. Diagnóstico de anemia e ocorrência de suplementação com sulfato ferroso.

\begin{tabular}{lccc}
\hline & Anêmicos & Não-anêmicos & $\begin{array}{c}\text { Em suplementação } \\
\text { com sulfato ferroso }\end{array}$ \\
\cline { 4 - 5 } Meninas & $\mathrm{N}(\%)$ & $\mathrm{N}(\%)$ & $\mathrm{N}(\%)$ \\
\cline { 1 - 1 } 3 a 4 anos e 11 meses & $3(23)$ & $10(77)$ & $8(61,54)$ \\
5 a 6 anos e 11 meses & $1(5)$ & $19(95)$ & $12(60,00)$ \\
Meninos & & & \\
\cline { 1 - 1 } 3 a 4 anos e 11 meses & $2(10,53)$ & $17(89,47)$ & $11(57,89)$ \\
5 a 6 anos e 11 meses & $3(21,43)$ & $11(78,57)$ & $9(64,28)$ \\
\hline
\end{tabular}
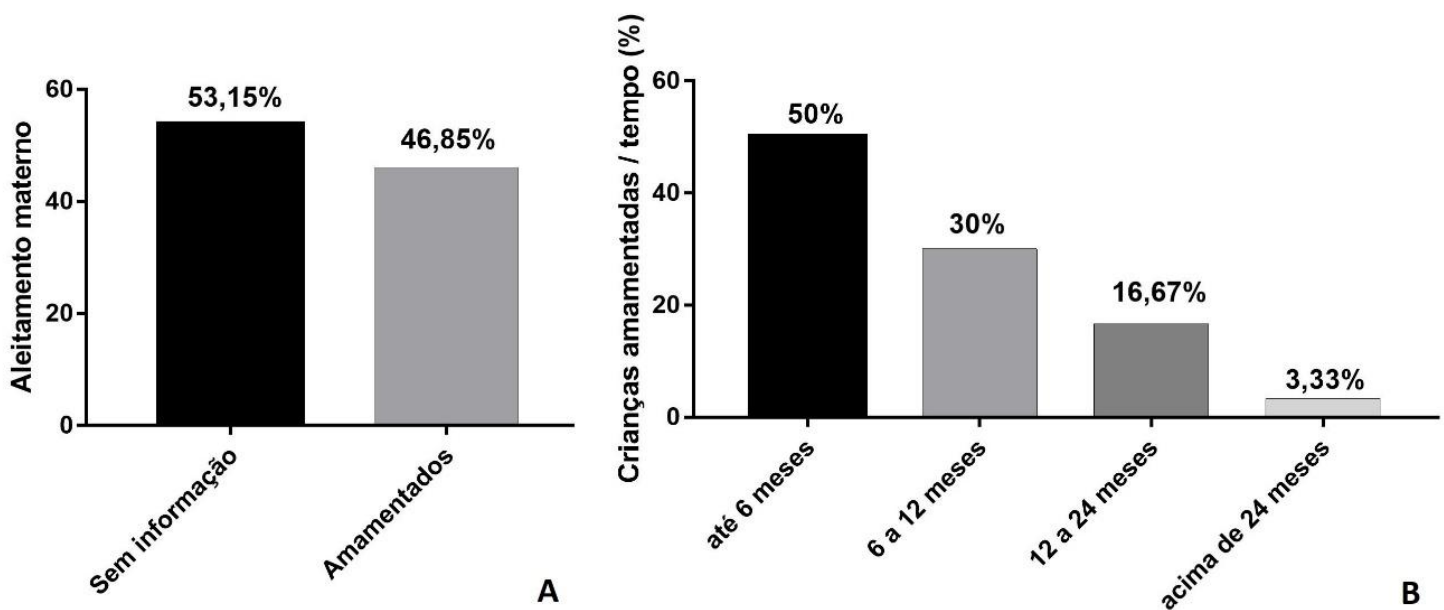

Figura 1. Frequência e tempo de aleitamento materno. $1 \mathrm{~A}$ - Informação sobre aleitamento materno no prontuário; 1B - Tempo de aleitamento materno indicado no prontuário.

Dentre as crianças com informação sobre o aleitamento materno exclusivo até os seis meses de vida, nota-se que em metade da população (50\%) essa atividade foi realizada ,entretanto, somente $16,67 \%$ prolongaram essa prática até os 24 meses, sendo ainda mais baixa a porcentagem de crianças amamentadas após esse período, contemplando somente 3,33\% (Figura 1).

Os dados antropométricos revelam que nenhuma criança se encontrava classificada com déficit de acordo com os indicadores analisados (P/E, E/I e $P / I)$ na idade de 5 anos a 6 anos e 11 meses (Tabela 3). Comparando-se com a faixa etária de 3 anos a 4 anos e 11 meses, poucas crianças se enquadraram nessa classificação $(15,15 \%)$. A presença de excesso de peso, avaliando-se o indicador de IMC/I, mostra maior prevalência de excesso de peso entre as meninas, quando comparado aos meninos.

Quanto a correlação entre o Índice de Massa Corporal (IMC) e possíveis alterações nos resultados de hemoglobina $(\mathrm{Hb})$ e hematócrito $(\mathrm{Ht})$, pode se observar (Figura 2) que não houve qualquer relação entre essas duas variáveis. 
Tabela 3. Indicadores antropométricos avaliados de acordo com o escore Z.

\begin{tabular}{|c|c|c|c|c|}
\hline \multirow{2}{*}{$\begin{array}{l}\text { Indicadores do estado } \\
\text { nutricional }\end{array}$} & \multicolumn{2}{|c|}{ Meninas } & \multicolumn{2}{|c|}{ Meninos } \\
\hline & 3 a 4 e 11 meses & 4 a 6 e 11 meses & 3 a 4 e 11 meses & 4 a 6 e 11 meses \\
\hline Déficit & $\mathrm{N}(\%)$ & $\mathrm{N}(\%)$ & $\mathrm{N}(\%)$ & $\mathrm{N}(\%)$ \\
\hline IMC/Idade & 0 & 0 & 0 & 0 \\
\hline Altura/Idade & $2(6,06)$ & $2(6,06)$ & 0 & 0 \\
\hline Peso/Idade & $1(3,03)$ & 0 & 0 & 0 \\
\hline Peso/Altura & 0 & 0 & 0 & 0 \\
\hline \multicolumn{5}{|l|}{ Adequado } \\
\hline IMC/Idade & $7(21,21)$ & $11(33,33)$ & $12(36,36)$ & $14(42,42)$ \\
\hline Altura/Idade & $11(33,33)$ & $18(54,54)$ & $18(54,54)$ & $15(45,45)$ \\
\hline Peso/Idade & $11(33,33)$ & $18(54,54)$ & $14(42,42)$ & $14(42,42)$ \\
\hline Peso/Altura & $8(24,24)$ & 0 & $11(33,33)$ & 0 \\
\hline \multicolumn{5}{|l|}{ Acima do adequado } \\
\hline IMC/Idade & $6(18,18)$ & $9(27,27)$ & $6(18,18)$ & $1(3,03)$ \\
\hline Altura/Idade & 0 & 0 & 0 & 0 \\
\hline Peso/Idade & $1(3,03)$ & $2(6,06)$ & $4(12,12)$ & $1(3,03)$ \\
\hline Peso/Altura & $5(15,15)$ & 0 & $6(18,18)$ & 0 \\
\hline
\end{tabular}

\section{DISCUSSÃO}

Atualmente a dosagem de ferritina sérica é o indicador mais sensível para a identificação da depleção dos estoques de ferro ${ }^{26}$. Os parâmetros mais específicos para a determinação do status do ferro são: ferro sérico, ferritina sérica, capacidade de ligação total do ferro, índice de saturação da transferrina, protoporfirina eritrocitária e, mais recentemente, o receptor de transferrina $^{27}$. Uma limitação do estudo foi a utilização apenas da concentração de hemoglobina como variável desfecho devido ao número reduzido de crianças com resultados de dosagem de ferritina ou transferrina sérica, capazes de expressar de forma mais acurada os níveis de ferro no organismo.

Apesar da baixa prevalência de anemia encontrada na população do presente estudo, vale ressaltar que os valores de $\mathrm{Hb}$, atualmente utilizados para o diagnóstico de anemia na atenção primária à saúde, podem subestimar a doença, uma vez que os níveis de $\mathrm{Hb}$ representam o último parâmetro a se alterar nessas condições. Comumente, a deficiência de ferro é resultado final de um longo período de balanço negativo, quando o nível de ferro corporal começa a cair, ocorre uma sequência de eventos até o esgotamento das reservas e, somente ao final, o conteúdo de ferro plasmático diminui e o fornecimento de ferro à medula torna-se insuficiente para a formação da hemoglobina ${ }^{26}$. Portanto, torna-se importante ressaltar que a deficiência de ferro se manifesta inicialmente pela redução da ferritina sérica, visto que esta representa a diminuição dos estoques de ferro, e deveria ser utilizado como indicador para diagnóstico precoce da anemia ferropriva.

Em um estudo realizado com grávidas adolescentes, no ambulatório de pré-natal para adolescentes da UNIFESP-EPM, foram analisadas as dosagens de $\mathrm{Hb}$ e ferritina, sendo observado que $17,9 \%$ das pacientes consideradas normais pelos valores da $\mathrm{Hb}$, apresentavam estoque de ferro depletado, quando avaliadas pela ferritina. Os resultados sugeriram que a dosagem de ferritina determinaria com maior precisão em relação a dosagem de $\mathrm{Hb}$, o distúrbio de ferro ${ }^{26}$. 


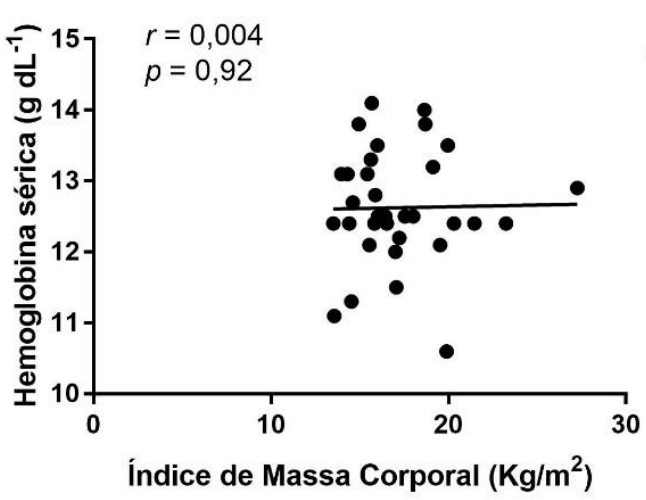

C

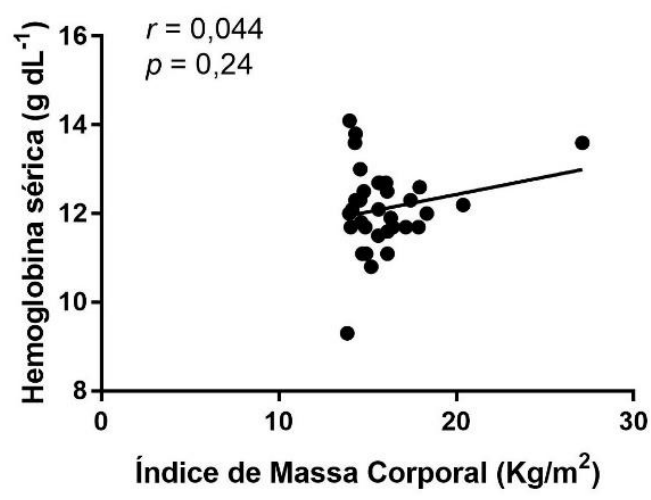

A

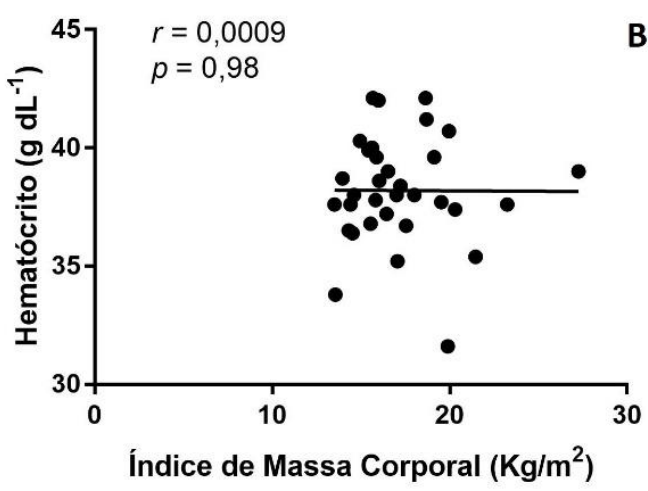

D

Figura 2. Correlação entre o Índice de Massa Corporal (IMC) e valores de hemoglobina ( $\mathrm{Hb}$ ) e hematócrito $(\mathrm{Ht})$. A - IMC $\times \mathrm{Hb}$ em meninas; B - IMC $\times \mathrm{Ht}$ em meninas; C - IMC $\times \mathrm{Hb}$ em meninos; D IMC $\times \mathrm{Ht}$ em meninos.

Na população estudada, a quantidade de crianças que recebem a suplementação de sulfato ferroso acima dos 24 meses, mesmo sem o diagnóstico definitivo de anemia ferropriva, representou mais de $50 \%$ do total de crianças, independente da faixa etária. Deve ser levado em consideração que a faixa etária para a suplementação profilática de anemia ferropriva é de 6 a 24 meses de idade ${ }^{19}$. Em um estudo que observou o efeito da suplementação de ferro na biodisponibilidade de zinco em ratos tratados com dietas específicas, observou-se que a biodisponibilidade desse nutriente foi reduzida quando associada a uma quantidade grande de ferro $^{28}$. A suplementação excessiva e além da idade recomendada para a profilaxia da deficiência de ferro pode intensificar a deficiência de outros nutrientes, como o zinco, visto que o consumo de ferro interage negativamente reduzindo a absorção do mesmo, prejudicando o estado nutricional da criança, uma vez que o zinco é um importante mineral para o desenvolvimento físico e neurológico ${ }^{27}$.

Em um estudo realizado em Teresina, que avaliou a efetividade da suplementação de sulfato ferroso em escolares, observou um aumento satisfatório na concentração de hemoglobina sérica em 114 crianças das 138 suplementadas com $40 \mathrm{mg}$ de sulfato ferroso (dose única) semanalmente ${ }^{30}$. Contudo, uma revisão sistemática de Cembranel, Dallazen e González-Chica ${ }^{31}$ buscou encontrar na literatura evidências da eficácia da suplementação de sulfato ferroso em crianças menores de 5 anos de idade e os resultados mostram que não há evidências na literatura científica de que estratégias de suplementação com sulfato ferroso, tanto com dose diária ou semanal, estejam relacionados com redução de anemia ferropriva $^{31}$.

A suplementação com sulfato ferroso, especialmente em crianças, tem ainda como limitação os efeitos colaterais associados aos compostos de ferro no lúmen intestinal, como diarréia, vômitos, náuseas e constipação. Nesse sentido, têm-se obtido maior efetividade na suplementação com ferro quelato, o qual, devido a sua ligação com aminoácido, provoca menos efeitos colaterais, aumentando a adesão ao tratamento ${ }^{32}$. 
Em um estudo com 790 crianças, de 6 a 36 meses realizado por Ribeiro e Sigulem ${ }^{33}$, foi utilizada a suplementação de ferro quelato bisglicinato e um fator limitante foi justamente a recusa na administração frequente da suplementação, sendo que mais de $50 \%$ do grupo se recusaram a consumir o suplemento justificando as intercorrências gastrointestinais e intolerância ao sulfato ferroso, assim apenas $30,8 \%$ dos pais ou responsável ofereceram regularmente a suplementação. Nesta população suplementada com ferro quelato não foram observados os efeitos colaterais (diarreia, constipação intestinal, vômitos ou náuseas) comuns do sulfato ferroso, contribuindo para a adesão total e continuidade do tratamento ${ }^{33}$.

Estudos mostram que a biodisponibilidade de ferro no sulfato ferroso é baixa quando comparada a absorção do bisglicinato de ferro, sendo este um suplemento mais eficiente no combate à anemia ferropriva, porém ainda não utilizado na atenção básica à saúde $^{34,35}$. Ribeiro e Sigulem ${ }^{33}$, puderam ainda observar ao decorrer de seu trabalho, o ganho de peso e estatura nas crianças suplementadas com bisglicinato de ferro e identificaram que a velocidade de crescimento da estatura foi significativamente maior no grupo suplementado com ferro quelato do que as do grupo controle, porém, não identificou o mesmo efeito no ganho de peso. Sendo assim, o estudo concluiu que a maior velocidade de crescimento entre os suplementados mostra que as crianças com bom estado nutricional, caracterizado pelo escore-Z, podem se beneficiar da suplementação de bisglicinato de ferro.

A ausência de aleitamento materno é apontada como fator de risco para a ocorrência de anemia ferropriva. Quando ofertado exclusivamente, o leite materno, apesar de sua baixa quantidade de ferro, apresenta alta biodisponibilidade deste nutriente ${ }^{36}$. A baixa duração do aleitamento materno exclusivo, cuja média de duração no Brasil é de 54,1 dias, assim como a introdução precoce de produtos processados, com característica de alta densidade energética e com baixa/intermediária biodisponibilidade de ferro na alimentação complementar da criança contribuem com o declínio dos níveis de $\mathrm{Hb}$ no primeiro ano de vida ${ }^{32}$, além de contribuir para o maior ganho ponderal.

Ao se avaliar a informação sobre aleitamento materno nos prontuários dos participantes do estudo, verificou-se que, além de a prática do aleitamento materno acontecer até os seis meses de vida em apenas metade das crianças cuja informação constava no prontuário, esse ato não foi prolongado até os dois anos ou mais, podendo ser considerado um fator importante para uma possível diminuição do estoque desse nutriente no organismo das crianças. Além disso, a ausência de informação sobre o aleitamento materno pela equipe de saúde apresenta-se como um fator de risco para a saúde das crianças, uma vez que não apenas a anemia ferropriva, mas diversas outras doenças estão associadas ao desmame precoce.

Alves et al. ${ }^{37}$ apontam que, para o correto diagnóstico de saúde da comunidade, é importante que as equipes de saúde na atenção básica estejam atentas ao surgimento de situações caracterizadas como de risco para o adoecimento e morte da população infantil. Destacam ainda que a avaliação da presença de situações de risco e vulnerabilidade deve constar na abordagem à saúde da criança desde a primeira consulta observando aspectos como problemas familiares e socioeconômicos; aleitamento materno ausente ou não exclusivo; não realização de vacinas; suspeita ou evidência de violência, entre outros ${ }^{37,38}$.

No presente estudo foi observada a ausência de informações no prontuário que pudessem caracterizar o grau de vulnerabilidade das crianças avaliadas, especialmente referente ao aleitamento materno, fator que pode levar ao negligenciamento de condições que requerem a intervenção da equipe de saúde da família.

A situação de crescimento e desenvolvimento da criança é o principal indicador de suas condições de saúde. O estado nutricional de uma criança é excelente indicador de sua saúde global e preditor da qualidade de vida, sendo assim, monitorar o crescimento e ganho ponderal permite avaliar se a criança está desenvolvendo adequadamente ${ }^{39,40}$. Verificou-se como estado nutricional mais frequente na população o excesso de peso em todas as faixas etárias, com valores mais elevados para as meninas. Esses dados se relacionam com o processo de transição nutricional em grupos de baixo nível socioeconômico de forma semelhante aos de maior renda. $O$ processo de transição nutricional em crianças e adolescentes tem sido estudado, inclusive com significância no sexo feminino em relação ao masculino, mostrando que houve um considerável declínio na 
prevalência de desnutrição e aumento dos casos de excesso de peso, especialmente nas crianças do sexo feminino ${ }^{41}$, confirmando assim os resultados obtidos nesse trabalho.

Diversos fatores contribuem para um quadro de insegurança alimentar e desnutrição na população infantil, tais como falta de informação e acesso aos serviços de saúde pelos pais, desmame precoce, introdução inadequada da alimentação complementar, consumo de alimentos de baixa densidade nutricional, afetando de maneira direta o desenvolvimento cognitivo, aumentando o risco de infecções e favorecendo 0 aparecimento de doenças crônicas $^{42}$. Embora os indicadores nacionais das últimas décadas mostrem uma redução na prevalência de desnutrição infantil, esta condição ainda se apresenta como um problema de saúde pública, especialmente no segmento de 0 a 5 anos de idade e em população em elevada vulnerabilidade. Assim, é fundamental o olhar da equipe de saúde para o público infantil com vistas a promover hábitos de vida saudáveis que colaborem para o estabelecimento e manutenção de um adequado estado nutricional, desenvolvimento e saúde das crianças.

O principal fator limitante para este estudo foi a falta de informações importantes nos prontuários das crianças, contribuindo para a falta de cuidados médicos adequados, visto que o prontuário é um instrumento essencial no cuidado à saúde na atenção básica.

Conclui-se que a prevalência de anemia ferropriva nessa população foi baixa, porém, o diagnóstico apenas pelos níveis de $\mathrm{Hb}$ sem valores de ferritina sérica é limitante para a identificação de deficiência de ferro. Identificouse suplementação com sulfato ferroso mesmo sem indicação clínica e fora da faixa etária de suplementação profilática, podendo este ferro do suplemento estabelecer interação negativa com outros nutrientes, acarretando em prejuízo do estado nutricional da criança. Pode-se concluir ainda que, nesta população, o Índice de Massa Corporal não mostrou correlação com os indicadores de anemia. Novos estudos são necessários para a identificação de determinantes antropométricos e nutricionais na ocorrência de anemia ferropriva em crianças de comunidades de baixo nível socioeconômico.

\section{AGRADECIMENTOS E CONFLITO DE INTERESSE}

Os autores agradecem a equipe da ESF João Domingos Netto e a Secretaria Municipal de
Saúde de Presidente Prudente pela viabilização deste trabalho.

Afirmamos não haver conflitos de interesse na execução do presente trabalho.

\section{REFERÊNCIAS}

1. Monte CMG. Desnutrição: um desafio secular à nutrição infantil. J Pediatr (Rio de Janeiro). 2000;76(3):285-97.

DOI:

https://doi.org/10.2223/JPED.165

2. Biscegli TS, Elysio CC, Romera J, Libeth J. Avaliação do estado nutricional e prevalência da carência de ferro em crianças frequentadoras de uma creche. Rev Paul Pediatr. 2006;24(4):323329. DOI: https://doi.org/10.1590/S010305822007000400007

3. Figueroa PD, Neves EMDA, Beltrão RDLFN, Oliveira DMM, Cunha DMAL. Estado nutricional de micronutrientes de crianças segundo características pessoais e das creches. Cad Saúde Col. 2016;24(4):468-77. DOI: https://doi.org/10.1590/1414$\underline{462 \times 201600040150}$

4. Melo KM, Cruz ACP, Figueiredo MFSB, Pinho DL. Influência do comportamento dos pais durante a refeição e no excesso de peso na infância. Esc Anna Nery, 2017;21(4):e20170102. DOI: https://doi.org/10.1590/2177-9465-ean2017-0102

5. Santos VDA, Castro MA, Fisberg M, Fisberg RM. Qualidade nutricional dos padrões alimentares de crianças: existem diferenças dentro e fora da escola? J Pediatr. 2017;93(1):47-57. DOI: https://doi.org/10.1016/i.jped.2016.03.008

6. Nilson A, Mabel, Correia M, Ludmila A V, Alves $M$ F. Consumo alimentar entre crianças brasileiras de dois a cinco anos de idade: Pesquisa Nacional de Demografia e Saúde (PNDS) 2006. Ciên Saúde Col. 2013;18(11):3369-77. DOI: https://doi.org/10.1590/S1413$\underline{81232013001100026}$

7. Rodrigues VC, Mendes BD, Gozzi A, Sandrini F, Santana RG, Matioli G. Deficiência de ferro, prevalência de anemia e fatores associados em crianças de creches públicas do oeste do Paraná, Brasil. Rev Nutr. 2011;24(3):407-20. DOI: https://doi.org/10.1590/S1415-

\section{$\underline{52732011000300004}$}


8. McLean E, Cogswell M, Egli I, Wojdyla D, de Benoist B. Worldwide prevalence of anaemia, WHO Vitamin and Mineral Nutrition Information System, 1993-2005. Public Heal Nutr. 2009;12(4):444-54. DOI: https://doi.org/10.1017/S1368980008002401

9. Lundström U. Iron deficiency anaemia. Gut. 1994;35(6):864-5. DOI: https://doi.org/10.1136/gut.35.6.864-c

10. Spinelli MGN, Marchioni DML, Souza JMP, Souza SB de, Szarfarc SC. Fatores de risco para anemia em crianças de 6 a 12 meses no Brasil. Rev Panam Salud Pub. 2005;17(2):84-91. DOI: https://doi.org/10.1590/S102049892005000200004

11. Stefanini MLR, Colli C, Lerner BR. Anemia e desnutrição em escolares da rede pública do município de Osasco, São Paulo, Brasil. Cad Saude Púb. 1995;11(3):439-447. DOI: https://doi.org/10.1590/S0102311X1995000300019

12. Assis AMO, Gaudenzi ED, Gomes G, Ribeiro RC, Szarfarc SC, Souza SB. Níveis de hemoglobina, aleitamento materno e regime alimentar no primeiro ano de vida. Rev Saúde Púb. 2004;38(4):543-51.

DOI:

https://doi.org/10.1590/S0034-

$\underline{89102004000400010}$

13. Dunn JOC, Mythen MG, Grocott MP. Physiology of oxygen transport. BJA Educ. 2016;16(10):341-8. DOI: https://doi.org/10.1093/bjaed/mkw012

14. Soliman AT, De Sanctis V, Yassin M, Adel A. Growth and Growth hormone-Insulin Like Growth Factor-I (GH-IGF-I) axis in chronic anemias. Acta Biomed. 2017; 88(1):101-11.

15. McCann JC, Ames BN. An overview of evidence for a causal relationship between iron deficiency during development and deficits in cognitive or behavioral function 1-3. Am J Clin Nutr. 2007;85:931-45. DOI: https://doi.org/10.1093/aicn/85.4.931

16. World Health Organization. Daily Iron Supplementation in Infants and Children. Geneva, Switzerland; 2016.
17. Allali S, Brousse V, Sacri AS, Chalumeau M. Anemia in children: prevalence, causes, diagnostic work-up, and long-term consequences. Expert Rev Hematol USA. 2017;10(11):1023-8. DOI:

https://doi.org/10.1080/17474086.2017.1354696

18. Ministério da Saúde. Programa Nacional de Suplementação de Ferro. Manual de Condutas Gerais. Brasília - DF, 2013.

19. Portal da Saúde. Programa Nacional de Suplementação de Ferro. Departamento de Atenção Básica. 2018. [acesso em: 14 mai 2019]. Disponível em: http://bvsms.saude.gov.br/bvs/publicacoes/man ual suplementacao ferro condutas gerais.pdf

20. Cembranel F, Corso ACT, González-Chica DA. Inadequacies in the treatment of iron deficiency anemia among children registered in the national program of iron supplementation in florianopolis, santa catarina, brazil. Texto contexto Enferm. 2017;26(2):e06310015. DOI: http://dx.doi.org/10.1590/0104$\underline{07072017006310015 .}$.

21. Cervato-Mancuso AM, Tonacio LV, Silva ER, Vieira VL. A atuação do nutricionista na Atenção Básica à Saúde em um grande centro urbano. Ciên Saúde Col. 2012;17(12):3289-300. DOI: http://dx.doi.org/10.1590/S1413-

\section{4}

22. França CJ, Carvalho VCHS. Estratégias de educação alimentar e nutricional na Atenção Primária à Saúde: uma revisão de literatura. Saúde Debate. 2017;41(114):932-48. DOI: http://dx.doi.org/10.1590/0103-1104201711421

23. Scherer F, Beneduzi VL. Perfil nutricional e prevalência de anemia ferropriva em crianças. ConScientiae Saúde. 2011;10(3):433-40. DOI: https://doi.org/10.5585/conssaude.v10i3.2497

24. BRASIL. Resolução no 466, de 12 de dezembro de 2012. Diretrizes e Normas Regulamentadoras de Pesquisas Envolvendo Seres Humanos. Conselho Nacional de Saúde. 2012.

25. Brasil. Ministério da Saúde. Departamento de Atenção Básica. Vigilância Alimentar e Nutricional. Curvas de Crescimento da Organização Mundial da Saúde - OMS. 2018. 
[acesso em: 21 mai 2019]. Disponível em: http://dab.saude.gov.br/portaldab/ape vigilancia alimentar.php?conteudo=curvas de cresciment o

26. Papa ACE, Furlan JP, Pasquelle $M$, Guazzelli $C A P$, Figueiredo MSD, Camano L et al. A anemia por deficiência de ferro na grávida adolescente: comparação entre métodos laboratoriais. Rev Bras Ginecol Obstet. 2003;25(10):731-8. DOI: http://dx.doi.org/10.1590/S0100-

\section{6 .}

27. Costa CMD, Brum IR, Lima ES. Anemia e marcadores séricos da deficiência de ferro em grávidas atendidas na rede pública municipal de Manaus, Amazonas, Brasil. Acta Amaz. 2009;39(4):901-5. DOI:

http://dx.doi.org/10.1590/S0044-

$\underline{59672009000400018}$

28. Pedrosa LFC, Cozzolino SMF. Efeito da suplementação com ferro na biodisponibilidade de zinco em uma dieta regional do nordeste do Brasil. Rev Saúde Púb. 1993;27(4):266-70. DOI: http://dx.doi.org/10.1590/S0034-

$\underline{89101993000400006 .}$.

29. Lobo AS, Tramonte VLC. Efeitos da suplementação e da fortificação de alimentos sobre a biodisponibilidade de minerais. Rev Nutr. 2004;17(1):107-13.

DOI: http://dx.doi.org/10.1590/S1415$\underline{52732004000100012}$

30. Santos MM, Nogueira NN, Diniz AS. Efetividade de diferentes estratégias de intervenção com ferro nos níveis de hemoglobina e ferritina em escolares de Teresina, Piauí, Brasil. Cad Saúde Púb. 2007;23(7):1547-52. DOI: http://dx.doi.org/10.1590/S0102311X2007000700005

31. Cembranel F, Dallazen C, Gonzalez-Chica D A. Efetividade da suplementação de sulfato ferroso na prevenção da anemia em crianças: revisão sistemática de literatura e metanálise. Cad Saúde Púb. 2013;29(9):1731-51.

DOI:

https://doi.org/10.1590/S0102

$\underline{311 \times 2013001300014}$

32. Duque X, Martinez H, Vilchis-Gil J. Effect of supplementation with ferrous sulfate or iron bisglycinate chelate on ferritin concentration in
Mexican schoolchildren: a randomized controlled trial. Nutr J. 2014;13:71. DOI: https://doi.org/10.1186/1475-2891-13-71

33. Ribeiro LC, Sigulem DM. Tratamento da anemia ferropriva com ferro quelato glicinato e crescimento de crianças na primeira infância. Rev Nutr. 2008;21(5):483-90. DOI: https://doi.org/10.1590/S1415$\underline{52732008000500001}$

34. Pineda O, Ashmead D. Effectiveness of treatment of iron-deficiency anemia in infants and young children with ferrous bis-glycinate chelate. Nutrition. 2001;17(5):381-4. DOI: https://doi.org/10.1016/S0899-9007(01)00519-6

35. Adelia CB, Fernando EV, Lindsay HA. Iron absorption from ferrous bisglycinate and ferric trisglycinate in whole maize is regulated by iron status. Am J Clin Nutr. 2000;71(6):1563-9. DOI: https://doi.org/10.1093/ajcn/71.6.1563.

36. Almeida N. O leite humano: qualidade e controle. Santos JR (org). Fisiologia e patologia da lactação. Natal: Ed. Sociedade Brasileira de Mastologia; 1995.

37. Alves CRL, Moulin ZS, Santos LC. Atenção à Saúde da Criança: aspectos básicos. Belo Horizonte; 2013.

38. Demarzo MMP, Oliveira CAD, Gonçalves DA. Prática clínica na Estratégia Saúde da Família: organização e registro. UNA-SUS: UNIFESP; 2017.

39. Giugliano R, Melo ALP. Diagnóstico de sobrepeso e obesidade em escolares: utilizando índice de massa corporal segundo padrão internacional. J Pediatr (Rio de Janeiro). 2004;80(2):129-34.

DOI:

https://doi.org/10.2223/1152

40. Abrantes MM, Lamounier JA, Colosimo EA. Prevalência de sobrepeso e obesidade em crianças e adolescentes das regiões Sudeste e Nordeste. J Pediatr (Rio de Janeiro). 2002;78(4):335-40. DOI: https://doi.org/10.1590/S0021$\underline{75572002000400014}$

41. Frota MA. Acompanhamento antropométrico de crianças: o ideal e o realizado. Rev Baiana Saúde Púb. 2014; 31(2):212. 
42. Victora CG, Adair L, Fall C, Hallal PC, Martorell $\mathrm{R}$, Richter $\mathrm{L}$, et al. Maternal and child undernutrition: consequences for adult health and human capital. Lancet 2008;371(9609):34057. DOI: https://doi.org/10.1016/S01406736(07)61692-4. 